\title{
Drosophila melanogaster positive transcriptional elongation factors regulate metabolic and sex-biased expression in adults
}

Haiwang Yang ${ }^{1 *}\left(\mathbb{D}\right.$, Denis Basquin ${ }^{2}$, Daniel Pauli ${ }^{2}$ and Brian Oliver ${ }^{1}$

\begin{abstract}
Background: Transcriptional elongation is a generic function, but is also regulated to allow rapid transcription responses. Following relatively long initiation and promoter clearance, RNA polymerase II can pause and then rapidly elongate following recruitment of positive elongation factors. Multiple elongation complexes exist, but the role of specific components in adult Drosophila is underexplored.

Results: We conducted RNA-seq experiments to analyze the effect of RNAi knockdown of Suppressor of Triplolethal and lilliputian. We similarly analyzed the effect of expressing a dominant negative Cyclin-dependent kinase 9 allele. We observed that almost half of the genes expressed in adults showed reduced expression, supporting a broad role for the three tested genes in steady-state transcript abundance. Expression profiles following lilliputian and Suppressor of Triplolethal RNAi were nearly identical raising the possibility that they are obligatory co-factors. Genes showing reduced expression due to these RNAi treatments were short and enriched for genes encoding metabolic or enzymatic functions. The dominant-negative Cyclin-dependent kinase 9 profiles showed both overlapping and specific differential expression, suggesting involvement in multiple complexes. We also observed hundreds of genes with sex-biased differential expression following treatment.

Conclusion: Transcriptional profiles suggest that Lilliputian and Suppressor of Triplolethal are obligatory cofactors in the adult and that they can also function with Cyclin-dependent kinase 9 at a subset of loci. Our results suggest that transcriptional elongation control is especially important for rapidly expressed genes to support digestion and metabolism, many of which have sex-biased function.
\end{abstract}

Keywords: lilli, AFF4, Su(Tpl), ELL, Cdk9, P-TEFb, SEC, Pausing

\section{Background}

Transcription by eukaryotic RNA polymerase II complex (RNAPII), composed of ten or more subunits $[1,2]$, involves multiple steps including initiation, promoter clearance, elongation, and termination. Each step requires different activities. The initiation process involves RNAPII recognition of core promoter elements at the transcription start site (TSS) [3]. Bound RNAPII opens a bubble in the duplex DNA and begins transcribing short RNAs by a

\footnotetext{
* Correspondence: haiwang.yang@nih.gov

${ }^{1}$ National Institute of Diabetes and Digestive and Kidney Diseases, National

Institutes of Health, 50 South Drive, Bethesda, MD 20892, USA

Full list of author information is available at the end of the article
}

scrunching mechanism [4]. While the first steps of initiation and promoter clearance are slow and highly regulated, elongation is rapid [5, 6]. This makes elongation an ideal mechanism for rapidly responding to changing conditions. A stalled or paused RNAPII has completed the slow steps such that de-pausing allows for rapid completion of transcription.

Great progress has been made on the biochemistry of elongation, although the exact composition of complexes is unclear due to biological context, biochemical complexity, and/or methodologies used in various studies. For the majority of genes, after transcription of about 20 to 50 nucleotides, RNAPII pauses when 5,6-dichloro-1- 
$\beta$-D-ribofuranosylbenzimidazole (DRB) sensitivity inducing factor (DSIF, a heterodimer of Suppressor of Ty 4 and 5 (Spt4 and Spt5)) and negative elongation factor (NELF) protein complexes bind to the RNAPII and transcript [7-9], to form a DSIF-NELF complex promoting the pausing of RNAPII [10-12]. Paused RNAPII is released by the positive transcription elongation factor $\mathrm{b}$ complex (P-TEFb), which phosphorylates the DSIF and NELF complexes as well as the carboxy-terminal domain of RNAPII (CTD) [13-17]. The catalytic and regulatory components of P-TEFb are Cyclin-dependent kinase 9 (Cdk9) and Cyclin $T$ respectively [18-20]. In addition to P-TEFb, Cdk9 can also form a complex with Cyclin $K$ [21]. Recruitment of P-TEFb kinase dissociates NELF, transforming DSIF into a positive elongation factor, triggering elongation [10].

$\mathrm{P}-\mathrm{TEFb}$ is often part of larger complexes (Table 1). For example, the super elongation complex (SEC), is a key regulator required for transcriptional elongation checkpoint control and rapid transcriptional induction [22-24]. In addition to P-TEFb, SEC contains 11-19 Lysine-rich Leukemia (ELL), encoded by Suppressor of Triplolethal $(\mathrm{Su}(\mathrm{Tpl}))$, and ALL1-Fused Gene From Chromosome 4(AF4)/Fragile mental retardation 2 (FMR2) family member 4 (AFF4), encoded by lilliputian (lilli) $[23,25,26]$. Another transcriptional elongation complex is the Dot1 complex (DotCom) [27], which contains the histone H3 Lys 79 (H3K79) methyltransferase DOT1L, encoded by grappa (gpp) [28]. H3K79 methylation by DOT1 may regulate transcription of genes by changing chromatin structure and accessibility of transcription factors [29], and has been implicated in many basic functions such as cell cycle regulation, chromosomal stability, and the DNA damage response [30]. There is also evidence that lilli and $\mathrm{Su}(\mathrm{Tpl})$ are essential for the H3K79 methylation function of DotCom during transcriptional activation, and evidence of large complexes including components of DOT1, P-TEFb, and SEC, such as the Elongation Assisting Protein complexes (EAP) and complexes of Mixed Lineage Leukemia 1 proteins (MLL)
[23, 27, 31, 32]. Additional complexity includes the Little Elongation Complex (LEC), which contains $\mathrm{Su}(\mathrm{Tpl})$ but lacks Lilli and Cdk9 [33], and AF4 family/ENL family/PTEFb complex (AEP), which contains Lilli and Cdk9 but lacks $\mathrm{Su}(\mathrm{Tpl})$ [34]. These studies indicate that there are many possible elongation complexes $[25,31]$. We have explored the roles of these complexes by examining the RNA expression profiles when some of these key components are altered in Drosophila. Specifically, knockdown of lilli or $\mathrm{Su}(\mathrm{Tpl}$ ) should disrupt SEC, EAP, and MLL. LEC should be altered by knockdown of $S u(T p l)$ but not lilli, and AEP should be altered by knockdown of lilli but not $\mathrm{Su}(\mathrm{Tpl})$. The dominant-negative form of $C d k 9$ should disrupt P-TEFb, SEC, and AEP, but not MLL.

The regulatory advantage of RNAPII pausing and elongation control is that rapid responses can be achieved if earlier rate-limiting steps occur, leaving only the more rapid elongation step for completion of transcription [5, 35-37]. Such a rapid transcriptional response is critical when organisms experience rapid environmental changes. For example, RNAPII pausing has been extensively studied in the context of the rapid transcription of Drosophila heat shock genes [38-40]. The rapid response is essential because failure to adjust to rapid temperature change results in catastrophic denaturation or mis-folding of proteins [41]. Increased levels of protein chaperones encoded by the heat shock loci ameliorate this stress. The presence of DSIF and NELF results in RNAPII pausing near the heat shock gene promoters [39], but following a heat shock, P-TEFb quickly redistributes to the heat shock genes, which allows RNAPII to elongate resulting in rapid production of transcripts [42, 43]. Other types of stress requiring a quick response in Drosophila, such as arbovirus infection, are also regulated at the level of elongation [44]. Regulation of transcription elongation also plays a role during development. An example is the pausing of RNAPII downstream of the sloppy-paired-1 promoter by NELF during the rapid blastoderm-stage of development [45]. It is likely that there are other genes responding rapidly due to

Table 1 Components of different elongation related complexes or protein group

\begin{tabular}{lcccll}
\hline Complex $^{\text {a }}$ & Lilli or AFF4 & Su(Tpl) or ELL & Cdk9 & Other components & References \\
\hline P-TEFb & - & - & + & Cyclin-T, Cyclin-K & [17, 93, 94] \\
SEC & + & + & $+{ }^{c}$ & AFF1, AF9, EAF, ENL & [23, 27] \\
DotCom & - & - & - & AF9, ENL, Dot1 & [27, 95] \\
EAP & + & + & + & AF5q31, AF9, ENL, Dot1 & {$[27,32,96]$} \\
MLL & + & + & - & AFF1, AF5q31, AF9, ENL & [23, 97-99] \\
LEC & - & + & - & EAF & [24, 33] \\
AEP & + & - & + & AF5q31, LAF4, ENL & [34] \\
\hline
\end{tabular}

${ }_{\mathrm{a}}^{\mathrm{P}-\mathrm{TEFb}}=$ Positive Transcript Elongation Factor b; SEC = Super Elongation Complex; DotCom = Dot1 Complex; EAP = Elongation Assisting Proteins; MLL = Mixed Lineage Leukemia 1 proteins; LEC = Little Elongation Complex; AEP = AF4 family/ENL family/P-TEFb complex

b" + " and "-" denote inclusion and exclusion of proteins respectively

${ }^{\mathrm{C}} \mathrm{A}$ few studies regard P-TEFb as an independent component of SEC [27, 100] 
normal physiology. Our expression profiles might help identify them.

Elongation control also plays a role in sexual development in Drosophila. Sex is determined by counting $\mathrm{X}$ chromosomes [46]. In wild-type flies, XX individuals are female and $\mathrm{XY}$ individuals are male. The genic imbalance created by $\mathrm{X}$ chromosome monosomy in males is compensated by increasing $\mathrm{X}$ chromosome gene expression [47]. At the regulatory level, the XX karyotype results in the activation of Sex-lethal $(S x l)$, which controls a major branch in the sex determination network [48]. Sxl regulates the splicing of transformer (tra) premRNA, which ultimately is responsible for nearly all sexually dimorphic aspects of development, physiology, and behavior [49-51]. One of the most important genes downstream of tra is doublesex $(d s x)$, which encodes sex-specific isoforms of a doublesex-mab3-related-transcription factor (DMRT). DMRTs are used to regulate sexual dimorphism in most metazoans [52]. The lilli and $\mathrm{Su}(\mathrm{Tpl})$ loci are likely to be direct targets of Dsx, and while the Cdk9 locus was not among the most obvious Dsx target genes, it has a strong and conserved Dsx binding site [51, 53]. Knocking down lilli or $\mathrm{Su}(\mathrm{Tpl})$ in $d s x$ expressing cells results in dramatic sex- and contextspecific phenotypes. For example, in males, the formation of specific first leg bristles known as sex combs, shows female pigmentation and narrower female-like morphology, but male distal/proximal orientation. In females, the first legs are normal, but bristles on the genitalia are missing. In males, the genitalia are normal. Thus, these genes function in female-specific development in one tissue and male-specific development in another [51]. If regulators of elongation play a post-developmental role in other aspects of sexual dimorphism, such as physiology, then we would expect to see sex-specific effects of disrupting the function of those complexes in adult flies.

\section{Results}

\section{Knockdown and overexpression efficiency}

We were interested in measuring the effect of lilli, $\mathrm{Su}(\mathrm{Tpl})$, and $\mathrm{Cdk} 9$ on gene expression in adults to probe for roles of elongation. Because these genes are required for organismal viability during development $[54,55]$, we bypassed the developmental defects by using ubiquitously expressed Gal4 [56] along with ubiquitously expressed temperature sensitive Gal80 repressor $\left(\right.$ Galso $\left.^{\text {ts }}\right)$ [57] and Gal4 responsive constructs expressing RNAi against lilli, and $S u(T p l)$ [58] (Fig. 1a). We also used a dominant negative $C d k 9$ construct $\left(C d k 9^{D N}\right)$ made by substituting an asparagine for an aspartic acid codon at position 199 (D199N) in the active site of the kinase. The same substitution has been used in other studies, where it has been shown to block P-TEFb CTD kinase activity [59, 60]. $C d k 9^{D N}$ under UAS control was introduced into flies using P-element mediated transformation. Expressing $C d k 9^{D N}$ during development resulted in lethality (DB and DP, unpublished data). Flies bearing the $C d k 9^{D N}$ transgene were viable to adulthood at $20{ }^{\circ} \mathrm{C}$ in the presence of both Gal4 and Gal8O ${ }^{t s}$.

We were concerned that interfering with these important transcriptional regulators would result in cell lethality and an uninformative signature of dying cells even if we bypassed developmental defects. To assess the effect of these genes on adult viability, we shifted 3 to 4 day old adult flies from the permissive temperature of $20{ }^{\circ} \mathrm{C}$ to the restrictive temperature of $29{ }^{\circ} \mathrm{C}$ to induce transgene expression. We observed no overt lethality after up to a week. The lack of adult lethality allowed us to measure the effect of $C d k 9$, lilli, and $S u(T p l)$ on the adult steady-state transcriptome in the absence of developmental defects or reduced viability. We crossed Gal4 and $G_{a l 8 O^{t s}}$ to the experimental lines and to two control lines, which acted as shams to monitor any differential expression due to Gal4 and Galso ${ }^{t s}$ alone (Fig. 1a). We used these sham genotypes without UAS transgenes as controls in addition to the temperature shifts. We collected sexed samples zero, one, and two days post temperature shift to $29{ }^{\circ} \mathrm{C}$. Flies were the same age posteclosion (7 days) when harvested. Because germline gene expression is not controlled in the expression system we used [61], we removed the gonads from the flies before preparing polyA+ RNA for stranded RNA-seq expression profiling. All experiments were biologically triplicated to quadruplicated using three flies per sample. We used a total of 99 samples, after confirming that mismatched replicate numbers did not impact conclusions (by bootstrapping, see Methods). We obtained above background RNA-seq reads for more than 8000 genes in each sample. We used the DESeq2 [62] Normalized Read Count statistical model to measure differential expression (see Methods). For each experimental sample, we made ratiometric measurements comparing replicate experimental samples to replicate sham samples as a reference. See Methods and GEO [63] (GSE77492) for detailed protocols, data, and replication statistics. A summary of all gene expression data can be found in the Additional file 1 .

We first assayed the effectiveness of the adult induction experiments (Fig. $1 \mathrm{~b}$ to $\mathrm{d}$ ). We detected expression of Gal4 and Galsots in all samples. Prior to the temperature shift, we observed no significant differential expression in lilli relative to shams (Fig. 1b), but we did observe modest but significant (Wald test, $p$ adj $<0.05$ is used throughout) reductions in target gene expression in the $\mathrm{Su}(\mathrm{Tpl})^{R N A i}$ flies (Fig. 1c). We also observed modest but significant overexpression of $C d k 9^{D N}$ (Fig. 1d), and we confirmed that the increased expression was from the transgenic $C d k 9^{D N}$ allele by measuring the fraction of the reads bearing the codon substitution $\left(\sim 84 \%\right.$ at $\left.20{ }^{\circ} \mathrm{C}\right)$. These data 


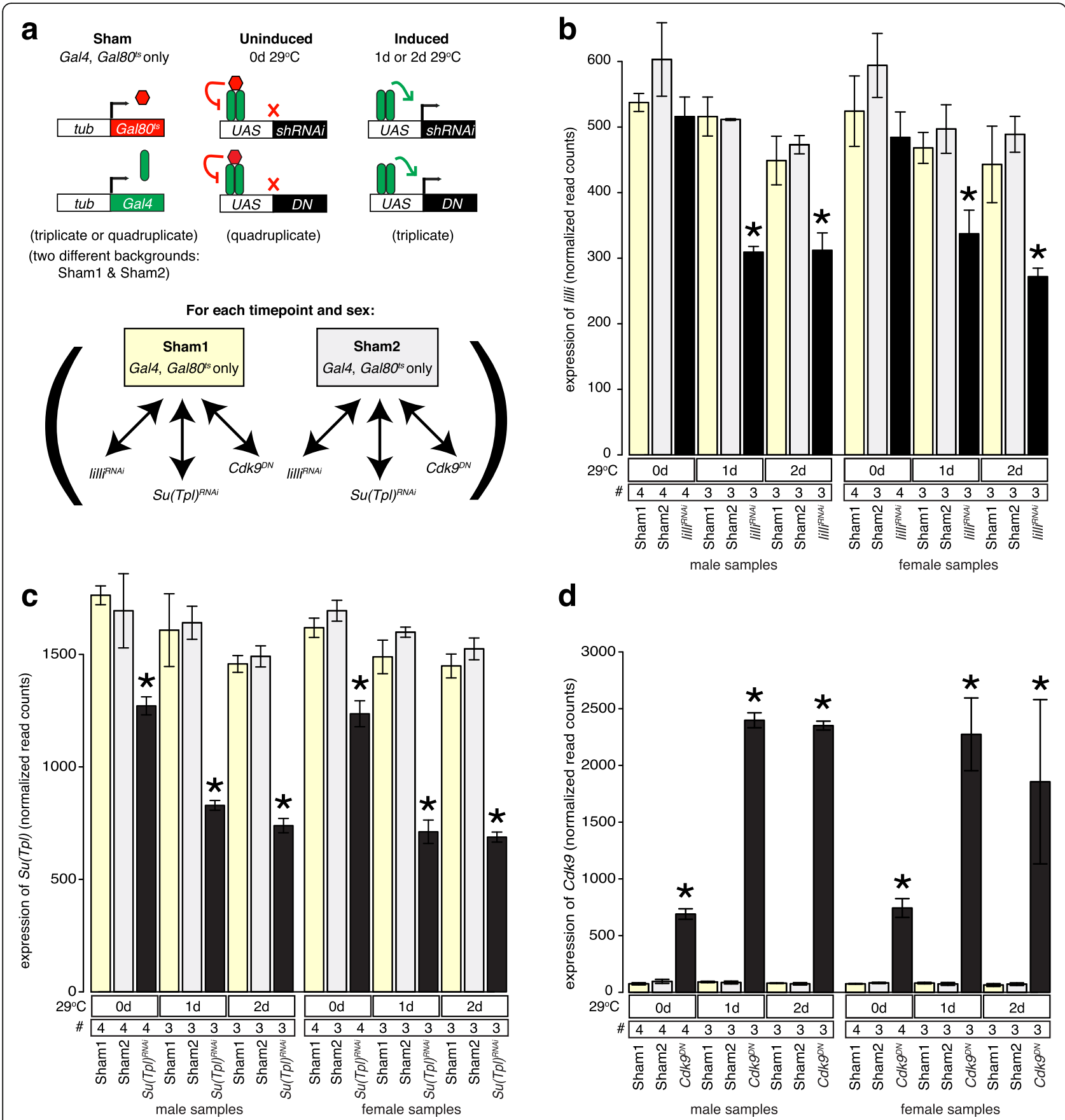

Fig. 1 Experimental design and efficiency of RNAi or $C d k 9^{D N}$ expression. a Experimental design. The cartoons (top) illustrate the sham genotypes where Gal80 $80^{\text {ts }}$ (red) and Gal4 (green) are produced in the absence of a responding UAS transgene (left), or when the UAS transgenes are present at the uninducing (middle) or inducing (right) temperatures. We used homozygous P\{tubP-GAL4\} ${ }^{L L 7}, P\left\{t u b P-G A L 80^{t 5}\right\}^{7}$ virgin females for all crosses. Sham flies were produced by crossing to lacZ (Sham1, yellow fill) or $w^{1118}$ (Sham2, gray fill) males. The schemata (bottom) shows how the shams were used as references for all induction timepoints for both females and males in results presented in subsequent figures. See Methods for further information. Transgenes are shown as bars, with regulatory sequences (open) from tubulin (tub) or the Gal4 upstream activation sequence (UAS), and encoding sequences (filled) from the Galso (red), Gal4 (green), shRNAi, and Cdk9 ${ }^{D N}$ (black) labeled. Gal80 (red hexagons), Gal4 (green ovals) proteins are shown. Active (bent black arrows) and repressed (red X) transcription are indicated. b-d Histograms showing expression of lilli (b), Su(Tpl) (c), and Cdk9 (d) transcripts based on normalized read counts (linear scale from DESeq2 [62]) across the gene models after zero (0d), one (1d), or two (2d) days of induction. Biological replicate numbers (\#) are indicated. Values for females (left) and males (right) are shown in each panel. Knockdown expression levels following induction of $l i l l i^{R N A i}$, Su(Tpl) $)^{R N A i}$, and $C d k 9^{D N}$ (black), and shams (yellow or gray) as well as one standard deviation (bars) are shown. Significant differential expression ( $p$ adj $<0.05$ from DESeq2) relative to sex and timepoint matched shams are shown (asterisk) 
indicate that the $S u(T p l)^{R N A i}$ and $C d k 9^{D N}$ transgenes were not fully "off" at $20^{\circ} \mathrm{C}$. Following the temperature shift to $29^{\circ} \mathrm{C}$, we observed dramatic reduction of $\mathrm{Su}$ ( Tpl) (Fig. 1c) and lilli (Fig. 1b) steady-state expression in the appropriate RNAi bearing flies and dramatic overexpression of $C d k 9^{D N}$ ( $\sim 96 \%$ from the DN allele; Fig. 1d) relative to shams. These data indicate that we altered the expression levels (and the encoded activity in the case of $C d k 9$ ) of the three genes under study.

\section{Effects on gene expression}

To explore the transcriptional responses to transgene induction, we compared replicated results from experimental samples relative to each of the two types of sham samples and asked for significantly differentially expressed genes in any of these time- and temperature-matched comparisons. We observed extensive and significant differences in the gene expression profiles relative to sham controls in these 36 pairwise comparisons, especially for reduced expression in the experimental samples (e.g., Fig. 2a, b). Overall we observed that 4792 genes showed significant differential expression in at least one pairwise comparison. This constituted $27 \%$ of all genes in the genome and $58 \%$ of the genes that we scored as expressed in all samples. To identify patterns that we could use to classify the responses of this large set of differentially expressed genes in a way that would allow us to infer which elongation complexes might be affected, we used kmeans to cluster the expression ratios of experimental relative to sham samples. We removed clusters driven by background genotypes (see Methods). We observed that 579 differentially expressed genes were clearly attributed to $l i l l i^{R N A i}, S u(T p l)^{R N A i}$, or $C d k 9^{D N}$ (Fig. 2c) treatments. There were four clusters of genes that were dominated by reduced gene expression on treatment: cluster 1 was down-regulated by $l i l l i^{R N A i}, S u(T p l)^{R N A i}$ and $C d k 9^{D N}$, clusters 2 and 3 were down-regulated following $\operatorname{lilli}^{R N A i}$ or $\mathrm{Su}(\mathrm{Tpl})^{R N A i}$ (cluster 3 genes were often up-regulated in $C d k 9^{D N}$ flies), and cluster 4 was down-regulated only following $C d k 9^{D N}$ expression. There was strong concordance between the $\operatorname{lilli}^{R N A i}$ and $S u(T p l)^{R N A i}$ profiles in all clusters. That there were no clear clusters of genes differentially expressed due to only one of these RNAi treatments, suggests that wild-type Lilli and $\mathrm{Su}(\mathrm{Tpl})$ positively regulate (directly or indirectly) many of the same genes. This is consistent with the hypothesis that these proteins are often obligatory components of the same functional complex. Cluster 1 showed reduced expression following $C d k 9^{D N}$ induction, suggesting that wild-type $l i l l i, S u(T p l)$, and $C d k 9$ positively regulate these genes (directly or indirectly), consistent with the participation of all three activities at a subset of genes. This is the correlation expected for disrupted SEC or EAP. Most gene expression altered by lilli and $S u(T p l)$ (cluster 2 and 3) were not regulated by
Cdk9, which is consistent with the composition of MLL. The $C d k 9^{D N}$ expression profile was distinct (cluster 4). Therefore, there is also $C d k 9$-specific differential expression profile, consistent with a role for P-TEFb alone.

\section{Expression of genes encoding enzymes}

To help determine if positive regulators of transcriptional elongation affect specific tissues or organs, we used FlyAtlas data from adults [64] to determine if differentially expressed genes had enriched expression in particular tissues. We found that the overall expression profile was not strongly enriched for genes expressed in any adult tissues (leftmost column of Fig. 3a), consistent with our use of the entire fly other than the gonads. Strikingly, the genes showing differential expression following $\operatorname{lilli}^{R N A i}, \operatorname{Su}(\mathrm{Tpl})^{R N A i}$, or $C d k 9^{D N}$ induction showed coherence in implied tissue-specificity (Fig. 3a). We found that all clusters with differentially expressed genes had enriched expression in the midgut. This is intriguing, as the midgut environment is subject to rapid changes as food passes through the digestive tract.

To determine if there was a functional category of genes differentially expressed due to the transgenes, we performed Gene Ontology (GO) term analysis (Fig. 3b) using expressed genes as the reference, and selected all terms that were enriched in one of the clusters. The enriched terms were associated with proteolysis, various aspects of amino acid or carbohydrate metabolism, transport, and pheromones. The genes regulated by all three of the transgenes (clusters 1 ) were enriched for genes encoding typsins with serine-type endopeptidase function (e.g., Serine protease 6). The genes down-regulated following $\operatorname{lilli}^{R N A i}$ or $\mathrm{Su}(\mathrm{Tpl})^{R N A i}$ but not $C d k 9^{D N}$ induction (clusters 2 and 3) were also enriched in genes encoding proteolysis functions in addition to metabolic, sensory, and transport functions. We show two examples of gene groups encoding important digestive enzymes that were down-regulated by transgene induction: the Lysosomal alpha-mannosidase (LMan) genes (Fig. 3c) and chymotrypsin encoding Jonah genes (Fig. 3d). We found that these genes required lilli and $S u(T p l)$ (but not $C d k 9$ ) for high level expression (clusters 2 and 3). They also both showed strongly midgutbiased expression in wild-type flies [64], and their wildtype functions involve carbohydrate hydrolysis in mannose production [65] and proteolysis (serine-type endopeptidase activity) [66] respectively.

We noticed that many of these enzyme-encoding genes with differential expression had short primary transcripts. Therefore, we determined if there was an overall tendency for preferential regulation of genes with short transcripts, by plotting the distributions of annotated primary transcript length of genes in each expression ratio cluster (Fig. 3e). Interestingly, the genes regulated by all three tested genes (cluster 1, see Fig. 2c) have significantly 


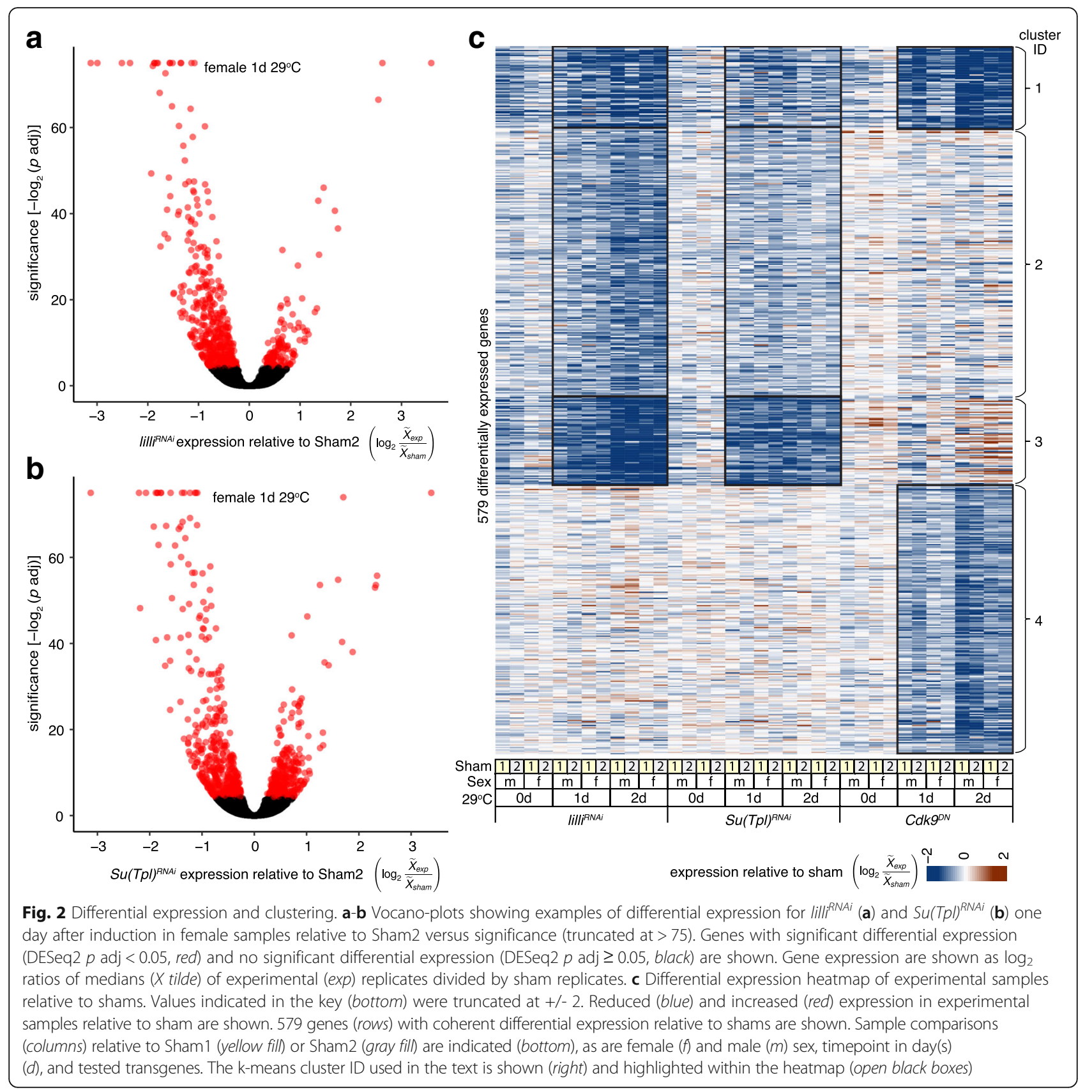

shorter primary transcripts than all expressed genes (1517 versus $2217 \mathrm{bp}$; Wilcoxon rank test, $\left.p=4 \times 10^{-6}\right)$. Moreover, the genes regulated by lilli and $\mathrm{Su}(\mathrm{Tpl}$ ) (cluster 2 and 3 , see Fig. 2c) also have significantly shorter primary transcripts than all expressed genes (1679 and 1027 versus $2217 \mathrm{bp}$; Wilcoxon rank test, $\left.p \leq 8 \times 10^{-6}\right)$. In contrast, the genes regulated by $C d k 9$ had insignificantly shorter primary transcript length compared to all expressed genes (2099 versus 2217 bp; Wilcoxon rank test, $p=0.6$ ). It is worth noting that the two clusters with the shortest primary transcripts (cluster 1 and 3) also had the strongest down regulation of expression after induction (Fig. 2c), as well as concordant enrichment of genes with proteolysis function (Fig. 3b). Given that control by elongation and short transcript length both contribute to rapid production of mature transcripts, these data suggest that many genes involved in metabolism have been optimized for rapid elongation.

\section{Sex-biased regulation}

We were intrigued by the role for positive elongation factors in the expression of a large number of metabolic enzyme encoding genes expressed in the midgut, as the Drosophila midgut shows sex-biased physiology and 


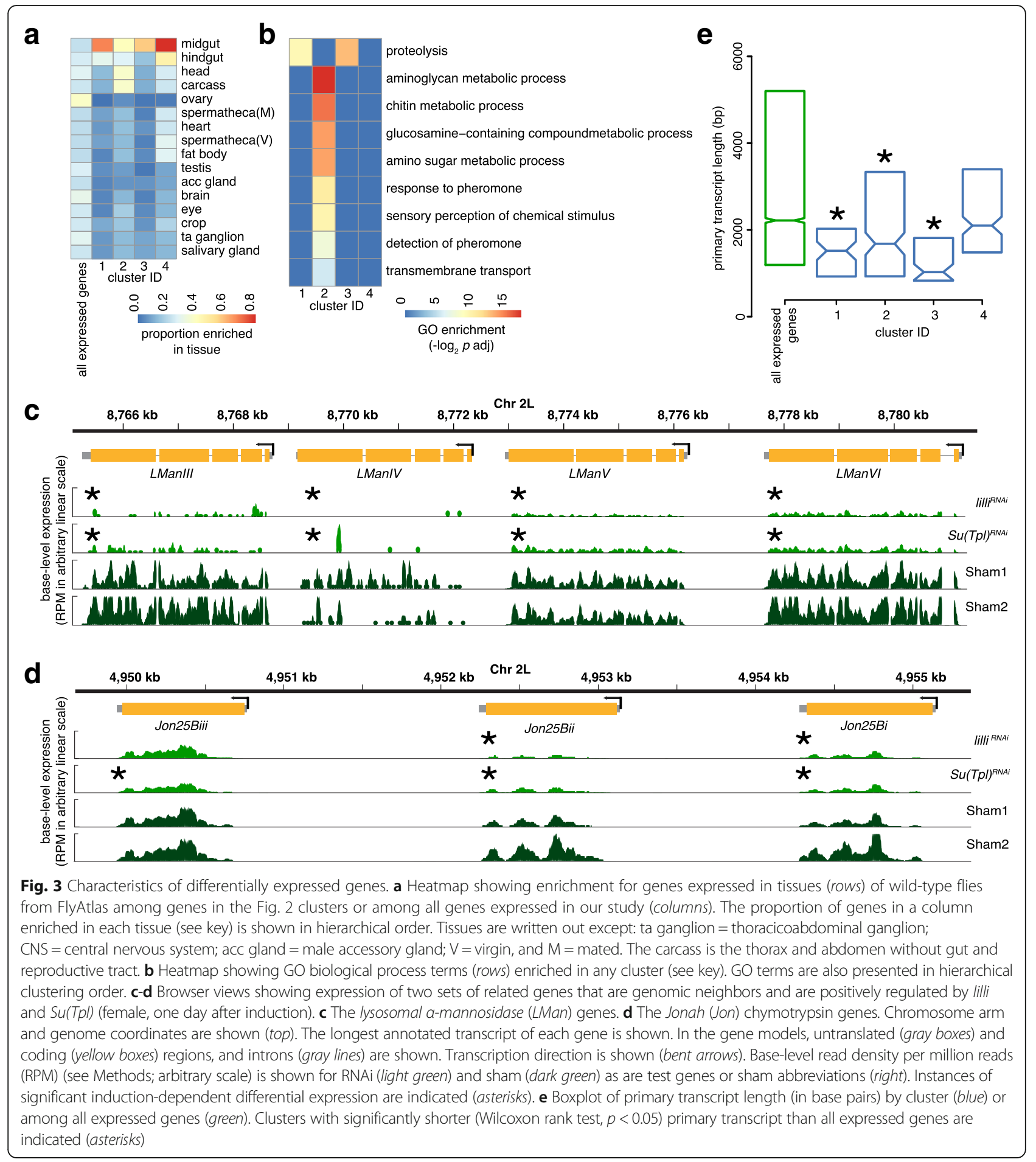

morphology, due to factors such as the immense metabolic requirements to support egg development and the requirements for specific saturation states in lipids used for pheromone production [67-69]. Additionally, lilli and $\mathrm{Su}(\mathrm{Tpl})$ are required for external sexual morphology [51]. This raised the possibility that there could be different roles for positive elongation factors in the sexes.
To explore this hypothesis, we measured sex-biased responses to gene expression treatments relative to shams for all 4792 differentially expressed genes. We then followed a k-means clustering procedure to identify genes with sex-biased patterns (Fig. 2c). We found three clusters (514 genes in total) showing a coherent sex difference in expression (Fig. 4a). Genes in cluster I showed higher 


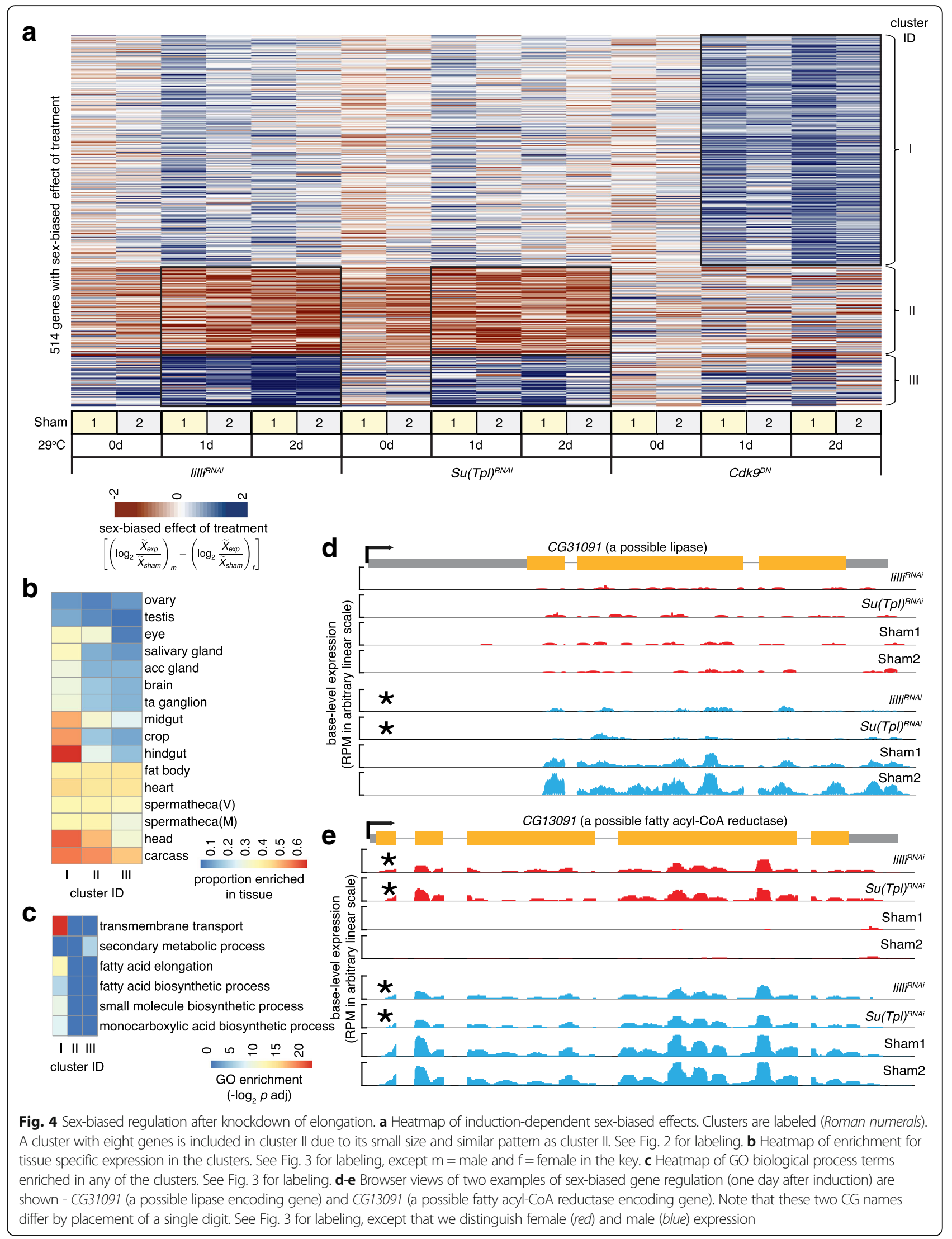


expression in males relative to females following $C d k 9^{D N}$ treatment, suggesting a role for P-TEFb. Genes in cluster II showed reduced expression in males relative to females, while those in cluster III showed higher expression in males relative to females following $l i l l i^{R N A i}$ and $S u(T p l)^{R-}$ ${ }^{N A i}$ treatments, but not $C d k 9^{D N}$. These results suggest a role for MLL in regulating sex-biased expression.

To determine where these genes with sex-biased responses might be expressed, we again compared to the FlyAtlas dataset (Fig. 4b). In addition to the digestive system, genes expressed in carcass were enriched in all three clusters. The FlyAtlas definition of carcass is the thorax and abdomen after removal of the other tissues and organs, so this category includes epidermis, musculature, and oenocytes, which perform liver-like functions in Drosophila [70]. We also found modest enrichment of genes expressed in a variety of non-gonadal tissues in the other clusters. To help determine the function of the genes showing sex-biased differential expression, we performed GO term enrichment analysis and observed that the most significant enrichment was trans-membrane transport and fatty acid elongation in genes regulated by Cdk9 (cluster I, Fig. 4c). Genes showing sex-biased expression due to lilli $^{R N A i}$ and $S u(T p l)^{R N A i}$ treatment were enriched in secondary metabolic processes (cluster III, Fig. 4c). Despite the lack of significant enrichment for GO terms in cluster II, we did observe sex-biased differential expression for at least a few genes encoding metabolic functions. For example, CG31091 encodes a protein with similarity to lipases and requires lilli and $\mathrm{Su}(\mathrm{Tpl})$ activity for its male-biased expression (cluster II, Fig. 4d). This possible lipase shows highly midgut-biased expression in wildtype flies [64]. As another example, CG13091 encodes a protein with similarity to fatty acyl-CoA reductases and low level of expression in females requires lilli and $\mathrm{Su}(\mathrm{Tpl})$ (cluster II, Fig. 4e). The putative reductase is highly expressed in wild-type fat body, heart, and carcass but not in other tissues [64]. Thus, like the overall pattern of differential expression, the sex-biased response to elongation factors was enriched in genes encoding metabolic functions.

\section{Discussion}

\section{Complex components}

Various positive elongation factor complexes have been described. Many of those complexes have overlapping components and complex composition is variable in the literature (Table 1) $[25,27,31]$. Therefore, genetic data can be useful for helping to determine the constituent components in biologically active complexes, or in epistatic relationships. For example, SEC contains both Lilli and $\mathrm{Su}(\mathrm{Tpl})$, while LEC and AEP contain either $\mathrm{Su}(\mathrm{Tpl})$ or Lilli (Table 1). In our expression profiles, lilli and $\mathrm{Su}(\mathrm{Tpl})$ positively regulate a nearly identical set of genes, which is consistent with them being obligatory cofactors in MLL, SEC, and EAP complexes. Our data does not support a role for LEC or AEP, although we cannot rule out the existence of these complexes. We also found that about $20 \%$ of the genes regulated by lilli and $\mathrm{Su}(\mathrm{Tpl})$ were also regulated by $C d k 9$. This is consistent with the presence of lilli, Su(Tpl), and Cdk9 in SEC and EAP [71]. However, we also found hundreds of genes positively regulated by $C d k 9$ only. The genes specifically regulated by $C d k 9$ might be dependent on $\mathrm{P}-\mathrm{TEFb}$ independently of the larger order complexes.

\section{Metabolic responses}

Promoter-proximal pausing of RNAPII is an interesting regulatory mechanism because it allows for rapid responses. RNAPII binding to the TSS, unwinding the DNA duplex and initiating transcription requires much more time in vivo $(10 \mathrm{~min}[72,73])$ than elongation (about $1.5 \mathrm{k}$ bases $(\mathrm{kb})$ per minute [74, 75]). Such rapid responses are especially important for heat shock, pathogen defense, and proper expression of some developmentally regulated genes [36, 39, 44, 45]. We found that positive elongation factors regulate many genes encoding metabolic enzymes, especially those expressed in the midgut. Release from pausing is an attractive mechanism for rapidly producing enzymes in response to feeding. Indeed, the Jonah genes encode chymotrypsins in the midgut and are expressed in feeding, but not in non-feeding stages of development, such as the first $12 \mathrm{~h}$ of adult life when flies are maturing and using residual fat body stores [76]. In mosquitos, the orthologous genes are rapidly induced in the gut by a blood meal $[77,78]$. We suggest that transcriptional elongation is an important regulator of rapid metabolic gene expression responses in adult Drosophila.

Rapid transcription also depends on gene length. Interestingly, genes positively regulated by the lilli and $\mathrm{Su}(\mathrm{Tpl})$ genes in our studies tend to have short transcripts, especially the ones with the highest level of differential expression after perturbation. For instance, most of the transcripts of chemotrypsin genes, such as Jonah genes and lambdaTry, are shorter than $1 \mathrm{~kb}$, which enables transcription in less than $40 \mathrm{~s}$. In general, the genes positively regulated by the lilli and $S u(T p l)$ genes produce even shorter transcripts than the heat shock genes (1751 versus $2231 \mathrm{bp}$ ). Although it may not be a prerequisite, short transcript length could be a feature evolutionarily favored by genes with rapid responses. We did not observe significantly shorter transcripts among genes regulated by $C d k 9$, suggesting that regulation of short transcripts is MLL-dependent.

\section{Sex-biased expression}

The lilli and $S u(T p l)$ loci contain many conserved and highly occupied Dsx binding sites, and driving $\operatorname{lilli}^{R N A i}$ 
or $S u(T p l)^{R N A i}$ in $d s x$ expressing cells results in specific switches in sexual dimorphism in the male first leg and the female genitalia, suggesting that these genes are required in both females and males, but in different locations [51]. A sex-biased role for Cdk9 has not been described, but the locus does have a conserved consensus Dsx binding site [51]. However, we do not know if this sex- and tissue-specific role is confined to morphology, or is more general. We found that sex-biased expression changed for about 200 genes due to $\operatorname{lilli}^{R N A i}$ or $\mathrm{Su}(\mathrm{Tpl})^{R N A i}$, and for about 300 genes due to $C d k 9^{D N}$. As was the case for morphology, the lilli and $S u(T p l)$ genes are required for both genes with female- and malebiased expression. This is consistent with the idea that sex-biased expression is highly context dependent and argues against strictly female- and male-biased batteries of genes that shows the same bias in all tissues. The $C d k 9$ regulated genes showed a more directional effect of reduced expression in females relative to males.

Many of the genes regulated by elongation factors encode metabolic functions. There is a great deal of precedent for sex-biased metabolism. For example, egg production is energy intensive and tightly linked to nutrient availability via insulin signaling [79]. At the morphological level, the Drosophila midgut is nearly twice as long in females as males and is regulated by the sexual identity pathway [67]. Finally, lipids and other metabolites also show dramatic sexual dimorphism [80]. Our work suggests that some of the sex-biased gene expression in metabolic pathways is due to indirect activity of Dsx target genes such as lilli and $\mathrm{Su}(\mathrm{Tpl})$.

\section{Conclusions}

Turning down positive elongation factors has a global transcriptional effect. Our data suggest that Lilli and $\mathrm{Su}(\mathrm{Tpl})$ are likely to be obligatory cofactors for the expression of many genes in soma, and that Cdk9 has both shared and $\mathrm{Lilli} / \mathrm{Su}(\mathrm{Tpl})$-independent functions. Our data also suggest that transcriptional elongation regulation by these factors is essential for rapid enzymatic and metabolic activities during digestion, a novel category of function regulated by rapid transcriptional elongation. In addition, the differential expression in females versus males suggests that positive elongation factors play roles in sexbiased gene regulation.

\section{Methods}

Summarized data is found in Additional file 1. A table of key resources is provided in Additional file 2, which describes the reagent or resources used; including chemical and biological reagents, critical commercial kits, genotypes of the flies, deposited data, and software. We also provided the sources and identifiers for these resources.

\section{Molecular genetics}

$C d k 9^{D N}$ transgenic lines were generated by PCRamplifying the $C d k 9$ open reading frame from $w^{1118}$ genomic DNA and cloned into pBluescript KS. Mutant $C d k 9^{D N}$, a substitution of amino acid 199 of the active site (aspartic acid to asparagine) was obtained by the megaprimer method [81] using a mutagenic oligonucleotide. The mutant DNA was cloned in the UASp vector [82] and injected in $w^{1118}$ embryos. A transgenic line with an unmapped insertion on the $3^{\text {rd }}$ chromosome was used in this study, but other lines show the same effect on development in the presence of various Gal4 drivers.

\section{Flies}

All flies were reared on the following media (per liter: $6.2 \mathrm{~g}$ agar, $59 \mathrm{~g}$ cornmeal, $59 \mathrm{~g}$ yeast, $5 \mathrm{ml}$ propionic acid and $26 \mathrm{ml} 99 \%$ methyl 4-hydroxybenzoate). Briefly, homozygous $w^{*} ;$ +; P\{tubP-GAL4 $\}^{L L 7}, P\left\{t u b P-G A L 80^{t s}\right\}^{7}$ virgin females were crossed to homozygous males with a UAS responsive transgene or homozygous males of control lines for shams. The paternal lines were $\operatorname{lilli}^{R N A i}$, $S u(T p l)^{R N A i}, C d k 9^{D N}, w^{1118}$, and lacZ. There are additional experiments in the GEO [63] series accession GSE77492 not presented here. First, we performed profiling following $g p p^{R N A i}$ but did not shown them in the results due to poor knockdown. Second, we used flies with an additional copy of $C d k 9^{D N}$, but observed a nonadditive effect of two copies of $C d k 9^{D N}$. Third, we ectopically expressed stand still, but these samples were for other purposes. All the parental lines were reared and crossed at $20{ }^{\circ} \mathrm{C}$, which also applied to their progeny. We collected adult progeny at 0 to $4 \mathrm{~h}$ post-eclosion, aged them for 5 to 7 days in fresh vials, and shifted to the restrictive temperature $\left(29^{\circ} \mathrm{C}\right)$ for 0,1 or 2 day(s). All the flies were 7 days old at the time of dissection.

\section{RNA-seq}

For each sample, three gonadectomized adults were dissected and crushed in 100 ul RNAlater solution (Life Technologies, Carlsbad CA) before sealing in deep 96-well plates at room temperature. Samples were homogenized with a mini-beadbeater (Biospec Products, Bartlesville OK) three times for $60 \mathrm{~s}$ each in the presence of 20 to 30 $1.0 \mathrm{~mm}$ glass beads per sample (BioSpec Products, Bartlesville OK). We extracted RNA using RNeasy according to the manufacturer (Qiagen, Valencia CA), except that we added $600 \mathrm{ul}$ RLT buffer and $700 \mathrm{ul} 70 \%$ ethanol in each well. We used $400 \mathrm{ng}$ total RNA in $50 \mathrm{ul}$ nuclease-free water (Quality Biological, Gaithersburg MD) for RNA-seq library preparation. We strictly followed a previously reported polyA+ strand-specific RNA-seq library preparation protocol [83]. We conducted singleend 50 bp RNA-seq on the HiSeq2000 Sequencing System (Illumina, San Diego CA). Reads were mapped to FlyBase 
[84] release 6.04 using STAR (v2.4.2a) with default parameters [85]. The overall agreement between biological replicates was $\geq 0.88$ (Pearson's $r$ ). We also mapped the reads of all samples to the intergenic regions, which we used as a measure of background expression noise for calculating the number of expressed genes. Genes expressed at $<95 \%$ percentile of intergenic expression were counted as unexpressed [86]. All samples with $29^{\circ} \mathrm{C}$ treatments were triplicated, and all samples without $29{ }^{\circ} \mathrm{C}$ treatments were quadruplicated except for female Sham2 samples (triplicated). To confirm that our results were robust to mismatched sample sizes, we used bootstrapping (100x) to randomly select three replicates among each sample set. The median number of re-identified differentially expressed genes was $96 \%$, suggesting that the variable samples sizes have little impact on our results. Therefore, we used all replicates in this work.

\section{Expression analysis}

For each sample, uniquely mapped read counts from HTSeq (v0.6.1p1) $(-$ stranded $=$ reverse) [87] were used as inputs in DESeq2 (v1.10.1). We used Normalized Read Counts as the unit of expression except for genome browser coverage plots, where we used base-level coverage of reads (generated by bedtools v2.25.0 [88]) normalized by RNA-seq library size in millions (RPM). For Normalized Read Counts, DESeq2 calculates the geometric mean $(\mathrm{GM})$ of each sample, and divides the raw read counts by the GM to obtain ratios of relative expression. For each sample, we used the median ratio as a samplespecific size factor ( 1 in our experiments), and normalized read counts were calculated as raw read counts divided by the size factor. Previous studies based 726 Drosophila individuals [86] demonstrated that the DESeq2 method was a high performing normalization approach relative to total counts, upper quartile, median, trimmed mean of M-values, quantile, reads per kilobase per million mapped reads, and un-normalized count data. We also used DESeq2 to calculate differential expression. For each experimental sample within the same sex and treatment, we compared experimental relative to each of the two sham genotypes. We retained genes with significantly differential expression (Wald test $p$ adj $<0.05$ ) in any of the comparisons. We further categorized these genes by their induction-dependent differential expression $\left(\log _{2}\right.$ ratio of the experiment expression relative to sham) across samples. We converted "N/A" values (divide by 0 ) to "0" (indicating no bias) for k-means clustering. We used the parameter $k=18$ (Fig. 2c) or 20 (Fig. 4a) based on evaluation of within-cluster sum of squares [89], and also confirmed that the major clusters were robust from $k=15$ to 25 . We used visual examination to remove clusters driven by differences between the two sham genotypes and clusters that showed weak differential expression, or responded even without induction.

We used FlyAtlas [64] microarray expression data to infer where differentially expressed genes were expressed in our experiments. We converted microarray Oligo IDs to FlyBase [84] gene IDs using DAVID (v6.7) [90]. Some genes have multiple microarray probes, and we calculated the mean signal using $1=$ "enriched", $0=$ "not different", and $-1=$ "depleted" as compared to the signal in the whole body. We defined enriched expression as mean $>0$. Gene Ontology (GO) [91] tests were conducted in FlyMine [92]. We used a Holm-Bonferroni cutoff of $p$ adj $<0.05$ for GO analysis and all tests were based on the background of expressed genes in any of our samples rather than all genes. We used the pheatmap package (v1.0.8) in $\mathrm{R}$ (v3.2.2) to generate all heat maps of tissue specificity and $\mathrm{GO}$ results.

\section{Additional files}

Additional file 1: Summary of gene expression and k-means clustering. (XLS $10240 \mathrm{~kb}$ )

Additional file 2: Key resources table. (XLS 55 kb)

\section{Abbreviations}

AEP: AF4 family/ENL family/P-TEFb complex; AF4: ALL1-Fused Gene From Chromosome 4; AFF4: AF4/FMR2 family member 4; Cdk9: Cyclin-dependent kinase 9; CTD: Carboxy-terminal domain of RNAPII; DN: Dominant negative; DotCom: Dot1 complex; DRB: 5,6-dichloro-1- $\beta$-D-ribofuranosylbenzimidazole; DSIF: DRB sensitivity inducing factor; EAP: Elongation assisting proteins; FMR2: Fragile mental retardation 2; GM: Geometric mean; GO: Gene ontology; Gpp: grappa; H3K79: Histone H3 Lys 79; LEC: Little Elongation Complex; Lilli: lilliputian; LMan: Lysosomal alpha-mannosidase; MLL: Mixedlineage leukemia 1 proteins; NELF: Negative elongation factor; PTEFb: Positive transcription elongation factor b complex; RNAPIl: RNA polymerase II; SEC: Super Elongation Complex; Su(Tpl): Suppressor of Triplolethal; TSS: Transcription start site

\section{Acknowledgements}

We are grateful to Cale Whitworth and Hangnoh Lee for help with RNA-seq library preparation, and Harold Smith and the NIDDK Genomics core for sequencing. This work utilized the computational resources of the $\mathrm{NIH}$ High-Performance Computing (HPC) Biowulf cluster (http://hpc.nih.gov). We would also like to thank Emily Clough for discussion and suggestions. This research was supported in part by the Intramural Research Program of the NIH, the National Institute of Diabetes and Digestive and Kidney Diseases (NIDDK).

\section{Funding}

This research was supported in part by the Intramural Research Program of the $\mathrm{NIH}$, the National Institute of Diabetes and Digestive and Kidney Diseases (NIDDK).

Availability of data and materials

GEO series accession (GSE77492)

\section{Authors' contributions}

$\mathrm{DP}$ and $\mathrm{BO}$ designed the experiment. DB, DP, and $\mathrm{BO}$ performed fly work and $\mathrm{HY}$ performed the genomics. $\mathrm{HY}$ and $\mathrm{BO}$ analyzed data and wrote the manuscript. All authors read and approved the final manuscript.

Competing interests

The authors declare that they have no competing interests.

Consent for publication

Not applicable. 


\section{Ethics approval and consent to participate}

Not applicable.

\section{Publisher's Note}

Springer Nature remains neutral with regard to jurisdictional claims in published maps and institutional affiliations.

\section{Author details}

${ }^{1}$ National Institute of Diabetes and Digestive and Kidney Diseases, National Institutes of Health, 50 South Drive, Bethesda, MD 20892, USA. ²Department of Genetics \& Evolution, Sciences III, University of Geneva, Boulevard d'Yvoy 4, CH 1205 Geneva, Switzerland.

\section{Received: 31 January 2017 Accepted: 3 May 2017} Published online: 18 May 2017

\section{References}

1. Myer VE, Young RA. RNA polymerase II holoenzymes and subcomplexes. J Biol Chem. 1998;273:27757-60.

2. Young RA. RNA polymerase II. Annu Rev Biochem. 1991:60:689-715.

3. Vannini A, Cramer P. Conservation between the RNA polymerase I, II, and III transcription initiation machineries. Mol Cell. 2012:45:439-46.

4. Revyakin A, Liu C, Ebright RH, Strick TR. Abortive initiation and productive initiation by RNA polymerase involve DNA scrunching. Science. 2006:314: 1139-43.

5. Jonkers I, Lis JT. Getting up to speed with transcription elongation by RNA polymerase II. Nat Rev Mol Cell Biol. 2015;16:167-77.

6. C Quaresma AJ, Bugai A, Barboric M. Cracking the control of RNA polymerase II elongation by 7SK snRNP and P-TEFb. Nucleic Acids Res. 2016;44:7527-39.

7. Min IM, Waterfall JJ, Core LJ, Munroe RJ, Schimenti J, Lis JT. Regulating RNA polymerase pausing and transcription elongation in embryonic stem cells. Genes Dev. 2011:25:742-54

8. Yamaguchi Y, Takagi T, Wada T, Yano K, Furuya A, Sugimoto S, Hasegawa J, Handa H. NELF, a multisubunit complex containing RD, cooperates with DSIF to repress RNA polymerase II elongation. Cell. 1999:97:41-51.

9. Missra A, Gilmour DS. Interactions between DSIF (DRB sensitivity inducing factor), NELF (negative elongation factor), and the Drosophila RNA polymerase II transcription elongation complex. Proc Natl Acad Sci U S A. 2010;107:11301-6.

10. Adelman K, Lis JT. Promoter-proximal pausing of RNA polymerase II: emerging roles in metazoans. Nat Rev Genet. 2012;13:720-31.

11. Wada T, Takagi T, Yamaguchi Y, Ferdous A, Imai T, Hirose S, Sugimoto S, Yano K, Hartzog GA, Winston F, et al. DSIF, a novel transcription elongation factor that regulates RNA polymerase II processivity, is composed of human Spt4 and Spt5 homologs. Genes Dev. 1998;12:343-56.

12. Wenzel S, Schweimer K, Rosch P, Wohrl BM. The small hSpt4 subunit of the human transcription elongation factor DSIF is a $\mathrm{Zn}$-finger protein with alpha/beta type topology. Biochem Biophys Res Commun. 2008;370:414-8.

13. Nonet $M$, Sweetser $D$, Young RA. Functional redundancy and structural polymorphism in the large subunit of RNA polymerase II. Cell. 1987;50:909-15.

14. Zehring WA, Lee JM, Weeks JR, Jokerst RS, Greenleaf AL. The C-terminal repeat domain of RNA polymerase II largest subunit is essential in vivo but is not required for accurate transcription initiation in vitro. Proc Natl Acad Sci U S A. 1988:85:3698-702.

15. Phatnani HP, Greenleaf AL. Phosphorylation and functions of the RNA polymerase II CTD. Genes Dev. 2006;20:2922-36.

16. Ping YH, Rana TM. DSIF and NELF interact with RNA polymerase ॥ elongation complex and HIV-1 Tat stimulates P-TEFb-mediated phosphorylation of RNA polymerase II and DSIF during transcription elongation. J Biol Chem. 2001;276:12951-8.

17. Peterlin BM, Price $\mathrm{DH}$. Controlling the elongation phase of transcription with P-TEFb. Mol Cell. 2006;23:297-305.

18. Mancebo HS, Lee G, Flygare J, Tomassini J, Luu P, Zhu Y, Peng J, Blau C, Hazuda D, Price D, Flores O. P-TEFb kinase is required for HIV Tat transcriptional activation in vivo and in vitro. Genes Dev. 1997:11:2633-44.

19. Yang X, Gold MO, Tang DN, Lewis DE, Aguilar-Cordova E, Rice AP, Herrmann $\mathrm{CH}$. TAK, an HIV Tat-associated kinase, is a member of the cyclin-dependent family of protein kinases and is induced by activation of peripheral blood lymphocytes and differentiation of promonocytic cell lines. Proc Natl Acad Sci U S A. 1997;94:12331-6.
20. Zhu Y, Pe'ery T, Peng J, Ramanathan Y, Marshall N, Marshall T, Amendt B, Mathews MB, Price $\mathrm{DH}$. Transcription elongation factor P-TEFb is required for HIV-1 tat transactivation in vitro. Genes Dev. 1997;11:2622-32.

21. Yu DS, Zhao R, Hsu EL, Cayer J, Ye F, Guo Y, Shyr Y, Cortez D. Cyclindependent kinase 9-cyclin $\mathrm{K}$ functions in the replication stress response. EMBO Rep. 2010;11:876-82.

22. Smith E, Lin C, Shilatifard A. The super elongation complex (SEC) and MLL in development and disease. Genes Dev. 2011;25:661-72.

23. Lin C, Smith ER, Takahashi H, Lai KC, Martin-Brown S, Florens L, Washburn MP, Conaway JW, Conaway RC, Shilatifard A. AFF4, a component of the ELL/ P-TEFb elongation complex and a shared subunit of MLL chimeras, can link transcription elongation to leukemia. Mol Cell. 2010;37:429-37.

24. Luo Z, Lin C, Shilatifard A. The super elongation complex (SEC) family in transcriptional control. Nat Rev Mol Cell Biol. 2012;13:543-7.

25. Chou S, Upton H, Bao K, Schulze-Gahmen U, Samelson AJ, He N, Nowak A, Lu H, Krogan NJ, Zhou Q, Alber T. HIV-1 Tat recruits transcription elongation factors dispersed along a flexible AFF4 scaffold. Proc Natl Acad Sci U S A. 2013:110:E123-31.

26. He N, Liu M, Hsu J, Xue Y, Chou S, Burlingame A, Krogan NJ, Alber T, Zhou Q. HIV-1 Tat and host AFF4 recruit two transcription elongation factors into a bifunctional complex for coordinated activation of HIV-1 transcription. Mol Cell. 2010:38:428-38.

27. Nguyen AT, Zhang Y. The diverse functions of Dot1 and H3K79 methylation. Genes Dev. 2011:25:1345-58.

28. Shanower GA, Muller M, Blanton JL, Honti V, Gyurkovics H, Schedl P. Characterization of the grappa gene, the Drosophila histone H3 lysine 79 methyltransferase. Genetics. 2005;169:173-84.

29. Farooq Z, Banday S, Pandita TK, Altaf M. The many faces of histone H3K79 methylation. Mutat Res Rev Mutat Res. 2016;768:46-52.

30. Kim W, Choi M, Kim JE. The histone methyltransferase Dot1/DOT1L as a critical regulator of the cell cycle. Cell Cycle. 2014;13:726-38.

31. Biswas D, Milne TA, Basrur V, Kim J, Elenitoba-Johnson KS, Allis CD, Roeder RG. Function of leukemogenic mixed lineage leukemia 1 (MLL) fusion proteins through distinct partner protein complexes. Proc Natl Acad Sci U S A. 2011; 108:15751-6.

32. Bonifer C, Cockerill PN. Transcriptional and Epigenetic Mechanisms Regulating Normal and Aberrant Blood Cell Development Preface. Transcriptional and Epigenetic Mechanisms Regulating Normal and Aberrant Blood Cell Development 2014;V-Vii.

33. Smith ER, Lin C, Garrett AS, Thornton J, Mohaghegh N, Hu D, Jackson J, Saraf A, Swanson SK, Seidel C, et al. The little elongation complex regulates small nuclear RNA transcription. Mol Cell. 2011:44:954-65.

34. Yokoyama A, Lin M, Naresh A, Kitabayashi I, Cleary ML. A higher-order complex containing AF4 and ENL family proteins with P-TEFb facilitates oncogenic and physiologic MLL-dependent transcription. Cancer Cell. 2010;17:198-212.

35. Core $L$, Lis JT. Transcription regulation through promoter-proximal pausing of RNA polymerase II. Science. 2008;319:1791-2.

36. Muse GW, Gilchrist DA, Nechaev S, Shah R, Parker JS, Grissom SF, Zeitlinger J, Adelman K. RNA polymerase is poised for activation across the genome. Nat Genet. 2007:39:1507-11.

37. Smith E, Shilatifard A. Transcriptional elongation checkpoint control in development and disease. Genes Dev. 2013:27:1079-88.

38. Zeitlinger J, Stark A, Kellis M, Hong JW, Nechaev S, Adelman K, Levine M, Young RA. RNA polymerase stalling at developmental control genes in the Drosophila melanogaster embryo. Nat Genet. 2007;39:1512-6.

39. Saunders A, Core $\sqcup$, Lis JT. Breaking barriers to transcription elongation. Nat Rev Mol Cell Biol. 2006;7:557-67.

40. Conaway JW, Shilatifard A, Dvir A, Conaway RC. Control of elongation by RNA polymerase II. Trends Biochem Sci. 2000;25:375-80.

41. Stetina T, Kostal V, Korbelova J. The Role of Inducible Hsp70, and Other Heat Shock Proteins, in Adaptive Complex of Cold Tolerance of the Fruit Fly (Drosophila melanogaster). PLoS One. 2015;10, e0128976.

42. Lis JT, Mason P, Peng J, Price DH, Werner J. P-TEFb kinase recruitment and function at heat shock loci. Genes Dev. 2000;14:792-803.

43. Shim EY, Walker AK, Shi Y, Blackwell TK. CDK-9/cyclin T (P-TEFb) is required in two postinitiation pathways for transcription in the C. elegans embryo. Genes Dev. 2002:16:2135-46.

44. Xu J, Grant G, Sabin LR, Gordesky-Gold B, Yasunaga A, Tudor M, Cherry S. Transcriptional pausing controls a rapid antiviral innate immune response in Drosophila. Cell Host Microbe. 2012;12:531-43.

45. Wang X, Lee C, Gilmour DS, Gergen JP. Transcription elongation controls cell fate specification in the Drosophila embryo. Genes Dev. 2007;21:1031-6. 
46. Erickson JW, Quintero JJ. Indirect effects of ploidy suggest X chromosome dose, not the X:A ratio, signals sex in Drosophila. PLoS Biol. 2007;5, e332.

47. Lucchesi JC, Kuroda MI. Dosage compensation in Drosophila. Cold Spring Harb Perspect Biol. 2015;7.

48. Hempel LU, Oliver B. Sex-specific DoublesexM expression in subsets of Drosophila somatic gonad cells. BMC Dev Biol. 2007;7:113.

49. Manoli DS, Foss M, Villella A, Taylor BJ, Hall JC, Baker BS. Male-specific fruitless specifies the neural substrates of Drosophila courtship behaviour. Nature. 2005:436:395-400.

50. Siwicki KK, Kravitz EA. Fruitless, doublesex and the genetics of social behavior in Drosophila melanogaster. Curr Opin Neurobiol. 2009;19:200-6.

51. Clough E, Jimenez E, Kim YA, Whitworth C, Neville MC, Hempel LU, Pavlou HJ, Chen ZX, Sturgill D, Dale RK, et al. Sex- and tissue-specific functions of Drosophila doublesex transcription factor target genes. Dev Cell. 2014;31:761-73.

52. Picard MA, Cosseau C, Mouahid G, Duval D, Grunau C, Toulza E, Allienne JF, Boissier J. The roles of Dmrt (Double sex/Male-abnormal-3 Related Transcription factor) genes in sex determination and differentiation mechanisms: Ubiquity and diversity across the animal kingdom. C R Biol. 2015;338:451-62.

53. Luo SD, Shi GW, Baker BS. Direct targets of the D. melanogaster DSXF protein and the evolution of sexual development. Development. 2011;138:2761-71.

54. Mummery-Widmer JL, Yamazaki M, Stoeger T, Novatchkova M, Bhalerao S, Chen D, Dietzl G, Dickson BJ, Knoblich JA. Genome-wide analysis of Notch signalling in Drosophila by transgenic RNAi. Nature. 2009;458:987-92.

55. Luschnig S, Moussian B, Krauss J, Desjeux I, Perkovic J, Nusslein-Volhard C. An F1 genetic screen for maternal-effect mutations affecting embryonic pattern formation in Drosophila melanogaster. Genetics. 2004;167:325-42.

56. Brand AH, Perrimon N. Targeted gene expression as a means of altering cell fates and generating dominant phenotypes. Development. 1993;118:401-15.

57. Ferris J, Ge H, Liu L, Roman G. G(o) signaling is required for Drosophila associative learning. Nat Neurosci. 2006;9:1036-40.

58. Perkins LA, Holderbaum L, Tao R, Hu Y, Sopko R, McCall K, Yang-Zhou D, Flockhart I, Binari R, Shim HS, et al. The Transgenic RNAi Project at Harvard Medical School: Resources and Validation. Genetics. 2015;201:843-52.

59. Garriga J, Segura E, Mayol X, Grubmeyer C, Grana X. Phosphorylation site specificity of the CDC2-related kinase PITALRE. Biochem J. 1996; 320(Pt 3):983-9.

60. Peng J, Marshall NF, Price DH. Identification of a cyclin subunit required for the function of Drosophila P-TEFb. J Biol Chem. 1998;273:13855-60.

61. Rorth P. Gal4 in the Drosophila female germline. Mech Dev. 1998;78:113-8

62. Love MI, Huber W, Anders S. Moderated estimation of fold change and dispersion for RNA-seq data with DESeq2. Genome Biol. 2014;15:550.

63. Edgar R, Domrachev M, Lash AE. Gene Expression Omnibus: NCBI gene expression and hybridization array data repository. Nucleic Acids Res. 2002;30:207-10.

64. Chintapalli VR, Wang J, Dow JA. Using FlyAtlas to identify better Drosophila melanogaster models of human disease. Nat Genet. 2007;39:715-20.

65. Chng WB, Bou Sleiman MS, Schupfer F, Lemaitre B. Transforming growth factor beta/activin signaling functions as a sugar-sensing feedback loop to regulate digestive enzyme expression. Cell Rep. 2014;9:336-48.

66. Ross J, Jiang H, Kanost MR, Wang Y. Serine proteases and their homologs in the Drosophila melanogaster genome: an initial analysis of sequence conservation and phylogenetic relationships. Gene. 2003;304:117-31.

67. Hudry B, Khadayate S, Miguel-Aliaga I. The sexual identity of adult intestinal stem cells controls organ size and plasticity. Nature. 2016;530:344-8.

68. Reiff T, Jacobson J, Cognigni P, Antonello Z, Ballesta E, Tan KJ, Yew JY, Dominguez $\mathrm{M}$, Miguel-Aliaga I. Endocrine remodelling of the adult intestine sustains reproduction in Drosophila. Elife. 2015;4, e06930.

69. Wicker-Thomas C, Henriet C, Dallerac R. Partial characterization of a fatty acid desaturase gene in Drosophila melanogaster. Insect Biochem Mol Biol. 1997:27:963-72.

70. Gutierrez E, Wiggins D, Fielding B, Gould AP. Specialized hepatocyte-like cells regulate Drosophila lipid metabolism. Nature. 2007;445:275-80.

71. Murali T, Pacifico S, Yu J, Guest S, Roberts 3rd GG, Finley Jr RL. DrolD 2011: a comprehensive, integrated resource for protein, transcription factor, RNA and gene interactions for Drosophila. Nucleic Acids Res. 2011;39:D736-43.

72. Lis J. Promoter-associated pausing in promoter architecture and postinitiation transcriptional regulation. Cold Spring Harb Symp Quant Biol. 1998;63:347-56.

73. Adelman K, Marr MT, Werner J, Saunders A, Ni Z, Andrulis ED, Lis JT. Efficient release from promoter-proximal stall sites requires transcript cleavage factor TFIIS. Mol Cell. 2005;17:103-12.
74. Yao J, Ardehali MB, Fecko CJ, Webb WW, Lis JT. Intranuclear distribution and local dynamics of RNA polymerase II during transcription activation. Mol Cell. 2007;28:978-90.

75. Ardehali MB, Lis JT. Tracking rates of transcription and splicing in vivo. Nat Struct Mol Biol. 2009;16:1123-4.

76. Carlson JR, Hogness DS. Developmental and functional analysis of Jonah gene expression. Dev Biol. 1985;108:355-68.

77. Edwards MJ, Lemos FJ, Donnelly-Doman M, Jacobs-Lorena M. Rapid induction by a blood meal of a carboxypeptidase gene in the gut of the mosquito Anopheles gambiae. Insect Biochem Mol Biol. 1997;27:1063-72.

78. Muller HM, Crampton JM, della Torre A, Sinden R, Crisanti A. Members of a trypsin gene family in Anopheles gambiae are induced in the gut by blood meal. EMBO J. 1993;12:2891-900.

79. Ikeya T, Galic M, Belawat P, Nairz K, Hafen E. Nutrient-dependent expression of insulin-like peptides from neuroendocrine cells in the CNS contributes to growth regulation in Drosophila. Curr Biol. 2002;12:1293-300.

80. Parisi M, Li R, Oliver B. Lipid profiles of female and male Drosophila. BMC Res Notes. 2011:4:198.

81. Kammann M, Laufs J, Schell J, Gronenborn B. Rapid insertional mutagenesis of DNA by polymerase chain reaction (PCR). Nucleic Acids Res. 1989;17:5404.

82. Thummel CS, Boulet AM, Lipshitz HD. Vectors for Drosophila P-elementmediated transformation and tissue culture transfection. Gene. 1988;74:445-56

83. Lee H, Cho DY, Whitworth C, Eisman R, Phelps M, Roote J, Kaufman T, Cook K, Russell S, Przytycka T, Oliver B. Effects of Gene Dose, Chromatin, and Network Topology on Expression in Drosophila melanogaster. PLoS Genet. 2016:12, e1006295.

84. Gramates LS, Marygold SJ, Santos GD, Urbano JM, Antonazzo G, Matthews BB, Rey AJ, Tabone CJ, Crosby MA, Emmert DB, et al. FlyBase at 25: looking to the future. Nucleic Acids Res. 2017;45:D663-71.

85. Dobin A, Davis CA, Schlesinger F, Drenkow J, Zaleski C, Jha S, Batut P, Chaisson M, Gingeras TR. STAR: ultrafast universal RNA-seq aligner. Bioinformatics. 2013;29:15-21.

86. Lin Y, Golovnina K, Chen ZX, Lee HN, Negron YL, Sultana H, Oliver B, Harbison ST. Comparison of normalization and differential expression analyses using RNA-Seq data from 726 individual Drosophila melanogaster. BMC Genomics. 2016;17:28.

87. Anders S, Pyl PT, Huber W. HTSeq-a Python framework to work with highthroughput sequencing data. Bioinformatics. 2015;31:166-9.

88. Quinlan AR, Hall IM. BEDTools: a flexible suite of utilities for comparing genomic features. Bioinformatics. 2010;26:841-2.

89. Witten DM, Tibshirani R. A framework for feature selection in clustering. J Am Stat Assoc. 2010;105:713-26.

90. da Huang W, Sherman BT, Lempicki RA. Systematic and integrative analysis of large gene lists using DAVID bioinformatics resources. Nat Protoc. 2009:4:44-57.

91. Ashburner M, Ball CA, Blake JA, Botstein D, Butler H, Cherry JM, Davis AP, Dolinski K, Dwight SS, Eppig JT, et al. Gene ontology: tool for the unification of biology. The Gene Ontology Consortium. Nat Genet. 2000;25:25-9.

92. Lyne R, Smith R, Rutherford K, Wakeling M, Varley A, Guillier F, Janssens H, Ji W, McLaren P, North P, et al. FlyMine: an integrated database for Drosophila and Anopheles genomics. Genome Biol. 2007:8:R129.

93. Kohoutek J. P-TEFb- the final frontier. Cell Div. 2009;4:19.

94. Lin X, Taube R, Fujinaga K, Peterlin BM. P-TEFb containing cyclin K and Cdk9 can activate transcription via RNA. J Biol Chem. 2002;277:16873-8.

95. Mohan M, Herz HM, Takahashi YH, Lin C, Lai KC, Zhang Y, Washburn MP, Florens L, Shilatifard A. Linking H3K79 trimethylation to Wnt signaling through a novel Dot1-containing complex (DotCom). Genes Dev. 2010;24:574-89.

96. Mueller D, Bach C, Zeisig D, Garcia-Cuellar MP, Monroe S, Sreekumar A, Zhou R, Nesvizhskii A, Chinnaiyan A, Hess JL, Slany RK. A role for the MLL fusion partner ENL in transcriptional elongation and chromatin modification. Blood. 2007;110:4445-54.

97. Rowley JD. The critical role of chromosome translocations in human leukemias. Annu Rev Genet. 1998;32:495-519.

98. Tenney K, Shilatifard A. A COMPASS in the voyage of defining the role of trithorax/MLL-containing complexes: linking leukemogensis to covalent modifications of chromatin. J Cell Biochem. 2005;95:429-36.

99. Huret IL, Dessen P, Bernheim A. An atlas of chromosomes in hematological malignancies. Example: 11q23 and MLL partners. Leukemia. 2001;15:987-9.

100. Dahlberg O, Shilkova O, Tang M, Holmqvist PH, Mannervik M. P-TEFb, the super elongation complex and mediator regulate a subset of non-paused genes during early Drosophila embryo development. PLoS Genet. 2015;11, e1004971. 